\title{
INFERENCE IN LÉVY-TYPE STOCHASTIC VOLATILITY MODELS
}

\author{
JEANNETTE H. C. WOERNER, * University of Göttingen
}

\begin{abstract}
Based on the concept of multipower variation we establish a class of easily computable and robust estimators for the integrated volatility, especially including the squared integrated volatility, in Lévy-type stochastic volatility models. We derive consistency and feasible distributional results for the estimators. Furthermore, we discuss the applications to time-changed CGMY, normal inverse Gaussian, and hyperbolic models with and without leverage, where the time-changes are based on integrated Cox-Ingersoll-Ross or Ornstein-Uhlenbeck-type processes. We deduce which type of market microstructure does not affect the estimates.
\end{abstract}

Keywords: Stochastic volatility model; Lévy process; statistical inference; power variation; integrated volatility; high-frequency data

2000 Mathematics Subject Classification: Primary 60J75; 60F05; 62M05

\section{Introduction}

Lévy-type stochastic volatility models formulated as time-changed Lévy processes have recently been introduced by Carr et al. (2003). These models can on the one hand be motivated by empirical observations, namely to merge the desirable properties of homogeneous exponential Lévy models and stochastic volatility models. Lévy-type stochastic volatility models incorporate a rich behaviour suitable to fit options across maturities, and make it possible to reproduce the time-dependence of the variance, the skewness and the kurtosis. Especially when taking an integrated mean reverting process as the time-change, this introduces volatility clustering to the model. Furthermore, it is straightforward to introduce leverage in the same way as in the Barndorff-Nielsen and Shephard model (cf. Barndorff-Nielsen and Shephard (2001)) by adding a mean process consisting of the process which drives the volatility.

On the other hand, these models can be motivated by the theoretical arguments that price processes for financial assets must have a jump component, but need not have a diffusion component (cf. Geman et al. (2001)). The argument may be summarized in the following way: all processes of interest may be expressed as a time-changed Brownian motion subordinated to a random clock, which may be viewed as a cumulative measure of economic activity. This clock must be certainly increasing and consist of jumps, since otherwise it would be locally deterministic. Finally these jumps in the clock introduce jumps in the price process.

Our aim is now to introduce a class of estimators for the integrated volatility, especially the integrated squared and the absolute volatility, which provides a measure for the level of volatility as needed for risk assessment and forecasting. Furthermore, the quantities of the squared and absolute integrated volatility are the basic quantities for pricing variance and volatility swaps,

Received 19 September 2006; revision received 5 March 2007.

* Postal address: Institut für Mathematische Stochastik, Universität Göttingen, Maschmühlenweg 8-10, D-37073

Göttingen, Germany. Email address: woerner@math.uni-goettingen.de 
cf. Howison et al. (2002). These financial instruments have become popular recently as a tool for hedging volatility risk.

We use the class of multipower variation estimators, i.e. estimators based on the appropriately normed sum of neighbouring log-returns, which lead to easily computable and robust estimators based on high-frequency data. For classical stochastic volatility models, i.e. when the driving process is a Brownian motion, this class of estimators was introduced by Barndorff-Nielsen and Shephard (2004) to estimate the $p$ th power of the integrated volatility for $p \geq 2$ robustly and was extensively studied in Barndorf-Nielsen and Shephard (2006), Barndorff-Nielsen et al. (2006b), and Woerner (2006). For the special case of bipower variation, this class of estimators was extensively studied in Barndorff-Nielsen et al. (2006a) and for the special case of power variation in Barndorff-Nielsen and Shephard (2003), and Woerner (2005). For the power variation case, Barndorff-Nielsen and Shephard (2007) and Corcuera et al. (2007) considered stable processes as driving processes, when the integral is viewed as an Itô integral or a refinement RiemannStieltjes integral, respectively. Woerner (2003a) considered general purely discontinuous Lévy processes and Corcuera et al. (2006) considered fractional Brownian motion.

The situation for purely discontinuous Lévy processes is, however, very different to the Brownian motion case. Heuristically this can be seen when comparing the results for nonnormed power variation in the classical setting

$$
\sum_{i}\left|\int_{t_{i-1}}^{t_{i}} \sigma_{s} \mathrm{~d} B_{s}\right|^{p} \stackrel{\mathrm{P}}{\rightarrow} \begin{cases}0 & \text { if } p>2 \\ \int_{0}^{t} \sigma_{s}^{2} \mathrm{~d} s & \text { if } p=2 \\ \infty & \text { if } p<2\end{cases}
$$

where $B$ denotes a Brownian motion and $\sigma$ a volatility process, and the case for the Lévy model

$$
\sum_{i}\left|L_{\int_{0}^{t_{i}} \sigma_{s} \mathrm{~d} s}-L_{\int_{0}^{t_{i}-1} \sigma_{s} \mathrm{~d} s}\right|^{p} \stackrel{\mathrm{P}}{\rightarrow} \begin{cases}\sum_{\infty}\left(\left|L_{\int_{0}^{u} \sigma_{s} \mathrm{~d} s}-L_{\int_{0}^{u-} \sigma_{s} \mathrm{~d} s}\right|^{p}: 0<u \leq t\right) & \text { if } p>\beta \\ \text { if } p<\beta,\end{cases}
$$

under appropriate regularity conditions (cf. Woerner (2003a)), where $\beta$ denotes the BlumenthalGetoor index of $L$, i.e. a measure for the activity of the jumps. Furthermore, from (1) we can see immediately that we can only derive results for power variation estimates of the integrated volatility for $p<\beta$ and not, as in the classical setting, for all $p$. As $\beta<2$, this implies that we cannot obtain results for the squared integrated volatility, i.e. the variance swap which corresponds to $p=2$. Power variation estimators in Lévy stochastic volatility models, when the model is not given by a time-change, as here, but by a stochastic integral with respect to a Lévy process, have been considered in Woerner (2003b). By using multipower variation estimates, where the number of neighbouring increments has to be chosen appropriately, we can also derive estimates for both the squared and the absolute integrated volatility.

The outline of this paper is as follows: first we introduce the notation, then we prove consistency and a distributional result for the multipower variation estimates. Furthermore, we discuss the robustness of the estimators and establish which type of mean process is negligible. This can be viewed as robustness against market microstructure noise or model misspecification. Finally, we apply our results to some specific stochastic volatility models. 


\section{Notation and definitions}

First let us now briefly review the definition of Lévy processes $X$, which are stochastic processes with independent and stationary increments. They are given by the characteristic function via the Lévy-Khinchin formula

$$
\mathrm{E}\left[\mathrm{e}^{i u X_{t}}\right]=\exp \left\{t\left(i \alpha u-\frac{\sigma^{2} u^{2}}{2}+\int\left(\mathrm{e}^{i u x}-1-i u h(x)\right) G(\mathrm{~d} x)\right)\right\},
$$

or for short by the Lévy triplet $\left(t \alpha, t \sigma^{2}, t G\right)_{h}$, where $\alpha$ denotes the drift, $\sigma^{2}$ the Gaussian part, $G$ the Lévy measure and $h$ is a truncation function, in general bounded and behaving like $x$ around the origin, hence ensuring the existence of the integral. Obviously $\sigma^{2}$ determines the continuous part and the Lévy measure determines the frequency and size of the jumps. If $\sigma=0$ and $\int(1 \wedge|x|) G(\mathrm{~d} x)<\infty$ the process has bounded variation, if $\int G(\mathrm{~d} x)<\infty$ the process jumps only finitely many times in any finite time-interval, called finite activity, it is a compound Poisson process. Furthermore, the support of $G$ determines the size and direction of the jumps. For more details see Sato (1999, pp. 148-159).

The concept of power variation in a mathematical framework was introduced in the context of studying the path behaviour of stochastic processes in the 1960s, cf. Berman (1965), Hudson and Mason (1976) for additive processes or Lepingle (1976) for semimartingales. Assume that we are given a stochastic process $X$ on some finite time interval $[0, t]$. Let $n$ be a positive integer and denote by $S_{n}=\left\{0=t_{n, 0}, t_{n, 1}, \ldots, t_{n, n}=t\right\}$ a partition of $[0, t]$, such that $0<t_{n, 1}<t_{n, 2}<\cdots<t_{n, n}$ and $\max _{1 \leq k \leq n}\left\{t_{n, k}-t_{n, k-1}\right\} \rightarrow 0$ as $n \rightarrow \infty$. Now the $p$ th power variation is defined to be

$$
\sum_{i=1}^{n}\left|X_{t_{n, i}}-X_{t_{n, i-1}}\right|^{p}=V_{p}\left(X, S_{n}\right)
$$

We are interested in the limit as $n \rightarrow \infty$, hence, the setting of high-frequency data. Closely related to the finiteness of this limit is the Blumenthal-Getoor index $\beta$, which provides a measure for the activity of the jumps. For Lévy processes it is defined by

$$
\beta=\inf \left\{\delta>0: \int_{-1}^{1}|x|^{\delta} G(\mathrm{~d} x)<\infty\right\} .
$$

It is well known that for $p>\beta, V_{p}$ tends to some nontrivial finite limit, whereas for $p<\beta$ the limit is zero. As in the Brownian motion case the natural step for getting nontrivial limits is to introduce an appropriate norming sequence, which leads to the normed $p$ th power variation introduced by Barndorff-Nielsen and Shephard (2003)

$$
\Delta_{n}^{\gamma} \sum_{i=1}^{n}\left|X_{t_{n, i}}-X_{t_{n, i-1}}\right|^{p}=\Delta_{n}^{\gamma} V_{p}\left(X, S_{n}\right)
$$

where $\gamma \in \mathbb{R}$ and $t_{n, i}-t_{n, i-1}=\Delta_{n}$ denotes the distance between neighbouring time-points in the equally spaced setting. A further extension of power variation is the concept of normed bipower or multipower variation, which makes it possible to also include the case for the squared or even higher-order integrated volatility. Let us introduce the following notation for the normed $(r+s)$ th bipower variation

$$
\Delta_{n}^{\gamma} \sum_{i=1}^{n-1}\left|X_{t_{n, i+1}}-X_{t_{n, i}}\right|^{r}\left|X_{t_{n, i}}-X_{t_{n, i-1}}\right|^{s}=\Delta_{n}^{\gamma} V_{r, s}\left(X, S_{n}\right),
$$


where $\gamma \geq 0$. Analogously for the $\sum_{i=1}^{k} r_{i}$ th $k$-power variation

$$
\Delta_{n}^{\gamma} \sum_{i=1}^{n-k+1} \prod_{j=i}^{i+k-1}\left|X_{t_{j}}-X_{t_{j-1}}\right|^{r_{j-i+1}}=\Delta_{n}^{\gamma} V_{r_{1}, \cdots, r_{k}}\left(X, S_{n}\right)
$$

where again $t_{n, i}-t_{n, i-1}=\Delta_{n}$.

\section{Multipower variation estimators}

As we have indicated in the introduction, multipower variation estimates, based on the appropriately normed multipower variation, are a natural tool for estimating integrated volatility. For models based on a time-changed Lévy process $k$-power variation estimates with $\sum_{i=1}^{k} r_{i}=p$ lead to estimates of $\int_{0}^{t} \sigma_{u}^{p / \beta} \mathrm{d} u$, where $\max _{i}\left(r_{i}\right)<\beta$; whereas for Lévy models involving integrals with respect to the Lévy process (cf. Woerner (2003b)) they estimate $\int_{0}^{t} \sigma_{u}^{p} \mathrm{~d} u$. This implies that in order to obtain an estimate for the absolute integrated volatility we need at least bipower variation and for the squared integrated volatility we need at least tripower variation, independent of the value of the Blumenthal-Getoor index.

The appropriate norming sequence is $\Delta_{n}^{1-\sum_{i=1}^{k} r_{i} / \beta}$, where $\beta$ denotes the Blumenthal-Getoor index of the underlying Lévy process $L$. Obviously the case $\beta=0$, e.g. a compound Poisson process, is excluded. This is due to the fact that we need the condition that around the origin the Lévy process behaves like a stable process with index $\beta$. The form of the norming sequence is in line with the Brownian motion case and the result for power variation of Lévy processes.

For simplicity we look at the bipower variation first.

Theorem 1. Let

$$
X_{t}=L_{\int_{0}^{t} \sigma_{s} \mathrm{~d} s}
$$

where L denotes a purely discontinuous Lévy process with Blumenthal-Getoor index $\beta \in(0,2)$. Assume that the density of the Lévy measure $g$ can be expanded in the following Taylor series expansion as $x \rightarrow 0$,

$$
g(x)=\frac{1}{|x|^{1+\beta}}\left(c_{0}+O(|x|)\right) .
$$

Assume, furthermore, that the Lévy triplet is given in the following form:

(a) if $\beta<1$, we take $h=0$ and the Lévy triplet is $(0,0, \operatorname{tg}(x))_{0}$,

(b) if $\beta \geq 1$ and $\int_{|x|>1}|x| g(x) d x<\infty$, we take $h(x)=x$ and the Lévy triplet is $(0,0, \operatorname{tg}(x))_{x}$,

(c) if $\beta \geq 1$ and $g$ is symmetric, we take

$$
h(x)=x 1_{|x| \leq 1}(x)
$$

and the Lévy triplet is $(0,0, g(x))_{x 1_{|x| \leq 1}(x)}$.

Assume that the process $\sigma$ is a locally bounded Riemann integrable, nonnegative, stochastically independent of $L$, and $\sigma^{r / \beta}$ and $\sigma^{s / \beta}$ have finite quadratic variation. Then for any $t>0$ and for $\max (r, s)<\beta$ we obtain

$$
\Delta_{n}^{1-(r+s) / \beta} V_{r, s}\left(X, S_{n}\right) \stackrel{\mathrm{P}}{\rightarrow} \mu_{r, \beta} \mu_{s, \beta}\left(\frac{c_{0}}{\beta}\right)^{(r+s) / \beta} \int_{0}^{t} \sigma_{u}^{(r+s) / \beta} \mathrm{d} u,
$$


as $n \rightarrow \infty$, where $\mu_{p, \beta}=\mathrm{E}\left(|S|^{p}\right)$ and $S$ is symmetrical $\beta$-stable distributed with Lévy triplet $\left(0,0, \beta|x|^{-1-\beta}\right)_{h}$.

We obtain the same result for $Z_{t}=Y_{t}+X_{t}$, when $Y_{t}$ is some stochastic process satisfying

$$
\begin{gathered}
\Delta_{n}^{1-(r+s) / \beta} V_{r, s}\left(Y, S_{n}\right) \stackrel{\mathrm{P}}{\rightarrow} 0, \\
\Delta_{n}^{1-(r+s) / \beta} \sum_{i=1}^{n-1}\left|X_{(i+1) \Delta_{n}}-X_{i \Delta_{n}}\right|^{r}\left|Y_{i \Delta_{n}}-Y_{(i-1) \Delta_{n}}\right|^{s} \stackrel{\mathrm{P}}{\rightarrow} 0, \\
\Delta_{n}^{1-(r+s) / \beta} \sum_{i=1}^{n-1}\left|Y_{(i+1) \Delta_{n}}-Y_{i \Delta_{n}}\right|^{r}\left|X_{i \Delta_{n}}-X_{(i-1) \Delta_{n}}\right|^{s} \stackrel{\mathrm{P}}{\rightarrow} 0 .
\end{gathered}
$$

Remark. As in the Brownian motion case, the absolute moments may be calculated explicitly (cf. Sato (1999, Example 25.10)), which leads to

$$
\mu_{p, \beta}=c_{\beta}^{p / \beta} \frac{2^{p} \Gamma((1+p) / 2) \Gamma(1-p / \beta)}{\sqrt{\pi} \Gamma(1-p / 2)},
$$

where $c_{\beta}=-2 \beta \Gamma(-\beta) \cos (\beta \pi / 2)$ if $\beta \neq 1$, and $c_{\beta}=\beta \pi$ if $\beta=1$.

Proof. First we prove the result for $Y=0$ and then show that under the conditions on $Y$ it is actually negligible. As a first step we calculate the characteristic function of the increment $X_{n, i}=X_{i \Delta_{n}}-X_{(i-1) \Delta_{n}}$, which is straightforward to obtain since $\sigma$ is independent of $L$,

$$
\begin{aligned}
& \mathrm{E}\left(\exp \left(i u\left(L_{\int_{0}^{i \Delta_{n}} \sigma_{s} \mathrm{~d} s}-L_{\int_{0}^{(i-1) \Delta_{n}} \sigma_{s} \mathrm{~d} s}\right)\right) \mid \sigma\right) \\
& \quad=\exp \left(\int_{(i-1) \Delta_{n}}^{i \Delta_{n}} \sigma_{s} \mathrm{~d} s \int\left(\mathrm{e}^{i u x}-1-i u h(x)\right) g(x) \mathrm{d} x\right) .
\end{aligned}
$$

Next we have to determine the distribution of $X_{n, i} / \Delta_{n}^{1 / \beta}$ conditionally under $\sigma$, for which we can use the condition on the Lévy measure and deduce that as $n \rightarrow \infty$ it behaves like a stable random variable. We obtain

$$
\begin{aligned}
\mathrm{E}\left(\exp \left(i u \frac{X_{n, i}}{\Delta_{n}^{1 / \beta}}\right) \mid \sigma\right)= & \exp \left(\int_{(i-1) \Delta_{n}}^{i \Delta_{n}} \sigma_{s} \mathrm{~d} s \int\left(\exp \left(\frac{i u x}{\Delta_{n}^{1 / \beta}}\right)-1-\frac{i u h(x)}{\Delta_{n}^{1 / \beta}}\right) g(x) \mathrm{d} x\right) \\
=\exp ( & \int_{(i-1) \Delta_{n}}^{i \Delta_{n}} \sigma_{s} \mathrm{~d} s \int\left(\mathrm{e}^{i u y}-1-\frac{i u h\left(\Delta_{n}^{1 / \beta} y\right)}{\Delta_{n}^{1 / \beta}}\right) \\
& \left.\times g\left(\Delta_{n}^{1 / \beta} y\right) \Delta_{n}^{1 / \beta} \mathrm{d} y\right) .
\end{aligned}
$$

Hence, as $n \rightarrow \infty$ the characteristic triplet of $X_{n, i} / \Delta_{n}^{1 / \beta}$ is

$$
\left(0,0, c_{0} \sigma_{n, i} \frac{1}{|x|^{1+\beta}}+O\left(\Delta_{n}^{1 / \beta}\right)\right)_{h},
$$

by the asymptotic expansion of $g$, where $\sigma_{n, i}=\int_{(i-1) \Delta_{n}}^{i \Delta_{n}} \sigma_{s} \mathrm{~d} s / \Delta_{n}$. This implies that asymptotically the scaled increments behave like a symmetric stable random variable with scale parameter $c_{0} \sigma_{n, i} / \beta$ and are independent conditionally under $\sigma$. 
Now we can use the law of large numbers for triangular schemes (cf. Gnedenko and Kolmogorov (1968, p. 134)) which states that for a triangular scheme of independent random variables, $\chi_{n, i}$, we obtain that in probability as $n \rightarrow \infty$

$$
\sum_{i=1}^{n} \chi_{n, i}-A_{n} \rightarrow 0,
$$

if

$$
\begin{gathered}
\sum_{i=1}^{n} \mathrm{P}\left(\left|\chi_{n, i}\right| \geq 1\right) \rightarrow 0, \\
\sum_{i=1}^{n} \mathrm{E}\left(\chi_{n, i}^{2} 1_{\left|\chi_{n, i}\right|<1}\right) \rightarrow 0, \\
A_{n}=\sum_{i=1}^{n} \mathrm{E}\left(\chi_{n, i}\right) .
\end{gathered}
$$

To get independent random variables we have to work conditionally under $\sigma$ and use the same technique as in Barndorff-Nielsen and Shephard (2004), namely considering independent copies $R=\sum_{i=1}^{n} \chi_{n, i}$ and $R^{\prime}=\sum_{i=1}^{n} \chi_{n, i}^{\prime}$. For the sum $R+R^{\prime}$, we can arrange the summands in an alternating way such there are two sums with independent random variables, which we denote by

$$
\tilde{\chi}_{n, i}=\Delta_{n}\left|\frac{\tilde{X}_{n, i+1}}{\Delta_{n}^{1 / \beta}}\right|^{r}\left|\frac{\tilde{X}_{n, i}}{\Delta_{n}^{1 / \beta}}\right|^{s} .
$$

For (7), taking $\delta>0$ to be sufficiently small, we obtain

$$
\begin{aligned}
\sum_{i=1}^{n-1} \mathrm{P}\left(\Delta_{n}\left|\frac{\tilde{X}_{n, i+1}}{\Delta_{n}^{1 / \beta}}\right|^{r}\left|\frac{\tilde{X}_{n, i}}{\Delta_{n}^{1 / \beta}}\right|^{s} \geq 1\right) & \leq \sum_{i=1}^{n-1} \Delta_{n}^{1+\delta} \mathrm{E}\left(\left.\left.|| \frac{\tilde{X}_{n, i+1}}{\Delta_{n}^{1 / \beta}}\right|^{r}\left|\frac{\tilde{X}_{n, i}}{\Delta_{n}^{1 / \beta}}\right|^{s}\right|^{1+\delta}\right) \\
& \rightarrow 0 .
\end{aligned}
$$

Similarly, for (8) we obtain

$$
\begin{aligned}
\sum_{i=1}^{n-1} \mathrm{E} & \left(\Delta_{n}^{2}\left|\frac{\tilde{X}_{n, i+1}}{\Delta_{n}^{1 / \beta}}\right|^{2 r}\left|\frac{\tilde{X}_{n, i}}{\Delta_{n}^{1 / \beta}}\right|^{2 s} 1_{\Delta_{n}\left|\tilde{X}_{n, i+1} / \Delta_{n}^{1 / \beta}\right|^{r}\left|\tilde{X}_{n, i} / \Delta_{n}^{1 / \beta}\right|^{s}<1}\right) \\
= & \sum_{i=1}^{n-1} \Delta_{n}^{1+\delta} \mathrm{E}\left(\left.\left.|| \frac{\tilde{X}_{n, i+1}}{\Delta_{n}^{1 / \beta}}\right|^{r}\left|\frac{\tilde{X}_{n, i}}{\Delta_{n}^{1 / \beta}}\right|^{s}\right|^{1+\delta}\right. \\
& \left.\times\left.\left.\Delta_{n}^{1-\delta}|| \frac{\tilde{X}_{n, i+1}}{\Delta_{n}^{1 / \beta}}\right|^{r}\left|\frac{\tilde{X}_{n, i}}{\Delta_{n}^{1 / \beta}}\right|^{s}\right|^{1-\delta} 1_{\Delta_{n}\left|\tilde{X}_{n, i+1} / \Delta_{n}^{1 / \beta}\right| r^{r}\left|\tilde{X}_{n, i} / \Delta_{n}^{1 / \beta}\right|^{s}<1}\right) \\
\leq & \sum_{i=1}^{n-1} \Delta_{n}^{1+\delta} \mathrm{E}\left(\left.\left.|| \frac{\tilde{X}_{n, i+1}}{\Delta_{n}^{1 / \beta}}\right|^{r}\left|\frac{\tilde{X}_{n, i}}{\Delta_{n}^{1 / \beta}}\right|^{s}\right|^{1+\delta}\right) \\
\rightarrow & 0 .
\end{aligned}
$$


To establish the limit in (9) we have to use the scaling relation for stable processes which leads to the sum $\sum_{i=1}^{n-1} \Delta_{n} \sigma_{n, i+1}^{r / \beta} \sigma_{n, i}^{s / \beta}$, which we have to show tends to $\int_{0}^{t} \sigma_{u}^{(r+s) / \beta} \mathrm{d} u$. For this, it is sufficient to show that

$$
\sum_{i=1}^{n-1} \Delta_{n} \sigma_{n, i+1}^{r / \beta} \sigma_{n, i}^{s / \beta}-\sum_{i=1}^{n-1} \Delta_{n} \sigma_{n, i}^{(r+s) / \beta} \stackrel{\mathrm{P}}{\rightarrow} 0
$$

We use Hölder's inequality and obtain

$$
\sum_{i=1}^{n-1} \Delta_{n} \sigma_{n, i}^{s / \beta}\left(\sigma_{n, i+1}^{r / \beta}-\sigma_{n, i}^{r / \beta}\right) \leq \Delta_{n}^{1 / 2}\left(\sum_{i=1}^{n-1} \Delta_{n} \sigma_{n, i}^{2 s / \beta}\right)^{1 / 2}\left(\sum_{i=1}^{n-1}\left(\sigma_{n, i+1}^{r / \beta}-\sigma_{n, i}^{r / \beta}\right)^{2}\right)^{1 / 2}
$$

where as $n \rightarrow \infty$,

$$
\sum_{i=1}^{n-1} \Delta_{n} \sigma_{n, i}^{2 s / \beta} \rightarrow \int_{0}^{t} \sigma_{u}^{2 s / \beta} \mathrm{d} u<\infty
$$

and

$$
\sum_{i=1}^{n-1}\left(\sigma_{n, i+1}^{r / \beta}-\sigma_{n, i}^{r / \beta}\right)^{2} \rightarrow\left[\sigma^{r / \beta}\right]_{t}<\infty,
$$

which yields (10). Hence, altogether, noting that for $\max (r, s)<\beta$ we have $\mathrm{E}|S|^{r}=\mu_{r, \beta}<\infty$ and $\mathrm{E}|S|^{s}=\mu_{s, \beta}<\infty$, we find that as $n \rightarrow \infty$,

$$
\tilde{A}_{n-1}=\sum_{i=1}^{n-1} \Delta_{n} \mathrm{E}\left(\left|\frac{\tilde{X}_{n, i+1}}{\Delta_{n}^{1 / \beta}}\right|^{r}\left|\frac{\tilde{X}_{n, i}}{\Delta_{n}^{1 / \beta}}\right|^{s}\right) \rightarrow\left(\frac{c_{0}}{\beta}\right)^{(r+s) / \beta} \mu_{r, \beta} \mu_{s, \beta} \int_{0}^{t} \sigma_{s}^{(r+s) / \beta} \mathrm{d} s .
$$

This yields $R+R^{\prime}-A_{n-1}-A_{n-1}^{\prime} \rightarrow 0$ and hence also $R-A_{n-1} \rightarrow 0$. Finally, we can deduce the unconditional result as for $\varepsilon>0$,

$$
\begin{aligned}
\lim _{n \rightarrow \infty} \mathrm{P}\left(\left|R-A_{n-1}\right| \geq \varepsilon\right) & =\lim _{n \rightarrow \infty} \mathrm{E}\left(1_{\left|R-A_{n-1}\right| \geq \varepsilon}\right) \\
& =\lim _{n \rightarrow \infty} \mathrm{E}\left(\mathrm{E}\left(1_{\left|R-A_{n-1}\right| \geq \varepsilon} \mid \sigma\right)\right) \\
& =0,
\end{aligned}
$$

which completes the result for $Y=0$.

For a nontrivial mean process we can use the same technique as in Woerner (2006), relying on a combination of triangular and Minkowski's inequality. For $0<r, s \leq 1$ we obtain, for all $\lambda>0$,

$$
\begin{aligned}
& \mathrm{P}\left(\left|\Delta_{n}^{1-(r+s) / \beta} \sum_{j=1}^{n-1}\right| X_{n, j+1}+\left.Y_{n, j+1}\right|^{r}\left|X_{n, j}+Y_{n, j}\right|^{s}\right. \\
& \left.\quad-\mu_{r, \beta} \mu_{s, \beta}\left(\frac{c_{0}}{\beta}\right)^{(r+s) / \beta} \int_{0}^{t} \sigma_{u}^{(r+s) / \beta} \mathrm{d} u \mid>\lambda\right) \\
& \quad \leq \mathrm{P}\left(\left|\Delta_{n}^{1-(r+s) / \beta} \sum_{j=1}^{n-1}\right| X_{n, j+1}+\left.Y_{n, j+1}\right|^{r}\left|X_{n, j}+Y_{n, j}\right|^{s}\right.
\end{aligned}
$$




$$
\begin{aligned}
& \left.-\Delta_{n}^{1-(r+s) / \beta} \sum_{j=1}^{n-1}\left|X_{n, j+1}\right|^{r}\left|X_{n, j}\right|^{s} \mid>\frac{\lambda}{2}\right) \\
& +\mathrm{P}\left(\left.\left|\Delta_{n}^{1-(r+s) / \beta} \sum_{j=1}^{n-1}\right| X_{n, j+1}\right|^{r}\left|X_{n, j}\right|^{s}\right. \\
& \left.\quad-\mu_{r, \beta} \mu_{s, \beta}\left(\frac{c_{0}}{\beta}\right)^{(r+s) / \beta} \int_{0}^{t} \sigma_{u}^{(r+s) / \beta} \mathrm{d} u \mid>\frac{\lambda}{2}\right) \\
& \leq \mathrm{P}\left(\left.\left|\Delta_{n}^{1-(r+s) / \beta} \sum_{j=1}^{n-1}\right| X_{n, j+1}\right|^{r}\left|Y_{n, j}\right|^{s} \mid>\frac{\lambda}{6}\right) \\
& +\mathrm{P}\left(\left.\left|\Delta_{n}^{1-(r+s) / \beta} \sum_{j=1}^{n-1}\right| Y_{n, j+1}\right|^{r}\left|X_{n, j}\right|^{s} \mid>\frac{\lambda}{6}\right) \\
& +\mathrm{P}\left(\left.\left|\Delta_{n}^{1-(r+s) / \beta} \sum_{j=1}^{n-1}\right| Y_{n, j+1}\right|^{r}\left|Y_{n, j}\right|^{s} \mid>\frac{\lambda}{6}\right) \\
& +\mathrm{P}\left(\left.\left|\Delta_{n}^{1-(r+s) / \beta} \sum_{j=1}^{n-1}\right| X_{n, j+1}\right|^{r}\left|X_{n, j}\right|^{s}\right. \\
& \left.\quad-\mu_{r, \beta} \mu_{s, \beta}\left(\frac{c_{0}}{\beta}\right)^{(r+s) / \beta} \int_{0}^{t} \sigma_{u}^{(r+s) / \beta} \mathrm{d} u \mid>\frac{\lambda}{2}\right) .
\end{aligned}
$$

As $n \rightarrow \infty$, (14) tends to zero by the first part of the proof and (11) to (13) tend to zero by the assumptions on $Y$.

For the other parameter constellations we obtain similarly, assuming without loss of generality that $r<s$ and $s>1$.

$$
\begin{gathered}
\mathrm{P}\left(\mid\left(\Delta_{n}^{1-(r+s) / \beta} \sum_{j=1}^{n-1}\left|X_{n, j+1}+Y_{n, j+1}\right|^{r}\left|X_{n, j}+Y_{n, j}\right|^{s}\right)^{1 / s}\right. \\
\left.-\left(\mu_{r, \beta} \mu_{s, \beta}\left(\frac{c_{0}}{\beta}\right)^{(r+s) / \beta} \int_{0}^{t} \sigma_{u}^{(r+s) / \beta} \mathrm{d} u\right)^{1 / s} \mid>\lambda\right) \\
\leq \mathrm{P}\left(\left|\left(\Delta_{n}^{1-(r+s) / \beta} \sum_{j=1}^{n-1}\left|X_{n, j+1}\right|^{r}\left|Y_{n, j}\right|^{s}\right)^{1 / s}\right|>\frac{\lambda}{6}\right) \\
+\mathrm{P}\left(\left|\left(\Delta_{n}^{1-(r+s) / \beta} \sum_{j=1}^{n-1}\left|Y_{n, j+1}\right|^{r}\left|X_{n, j}\right|^{s}\right)^{1 / s}\right|>\frac{\lambda}{6}\right) \\
+\mathrm{P}\left(\left|\left(\Delta_{n}^{1-(r+s) / \beta} \sum_{j=1}^{n-1}\left|Y_{n, j+1}\right|^{r}\left|Y_{n, j}\right|^{s}\right)^{1 / s}\right|>\frac{\lambda}{6}\right) \\
+\mathrm{P}\left(\mid\left(\Delta_{n}^{1-(r+s) / \beta} \sum_{j=1}^{n-1}\left|X_{n, j+1}\right|^{r}\left|X_{n, j}\right|^{s}\right)^{1 / s}\right.
\end{gathered}
$$




$$
\left.-\left(\mu_{r, \beta} \mu_{s, \beta}\left(\frac{c_{0}}{\beta}\right)^{(r+s) / \beta} \int_{0}^{t} \sigma_{u}^{(r+s) / \beta} \mathrm{d} u\right)^{1 / s} \mid>\frac{\lambda}{2}\right) .
$$

These expressions tend to zero under the same conditions as for the case above, which completes our proof.

However, for the squared integrated volatility, bipower variation is not sufficient, we have to look at tripower variation. Next we extend the result to $k$-power variation.

Theorem 2. Under the same conditions as in the previous theorem, for any $t>0$ and $\max _{i \in\{1, \cdots, k\}}\left(r_{i}\right)<\beta$, we obtain

$$
\Delta_{n}^{1-\left(\sum_{i=1}^{k} r_{i}\right) / \beta} V_{r_{1}, \ldots, r_{k}}\left(X, S_{n}\right) \stackrel{\mathrm{P}}{\rightarrow} \prod_{1 \leq i \leq k} \mu_{r_{i}, \beta}\left(\frac{c_{0}}{\beta}\right)^{\left(\sum_{i=1}^{k} r_{i}\right) / \beta} \int_{0}^{t} \sigma_{u}^{\left(\sum_{i=1}^{k} r_{i}\right) / \beta} \mathrm{d} u,
$$

as $n \rightarrow \infty$, where $\mu_{p, \beta}=\mathrm{E}\left(|S|^{p}\right)$ and $S$ is symmetrical $\beta$-stable distributed with Lévy triplet $\left(0,0, \beta|x|^{-1-\beta}\right)_{h}$.

Proof. The proof follows the same lines as the proof of Theorem 1. For the mean process $Y$ we have to consider all permutations of $X$ and $Y$ increments, however, it turns out that this condition is satisfied for the same processes as for the $k=2$ case.

Now we have to look at the conditions of Theorem 1. First we will see that the condition on the Lévy process is quite mild.

Example. (Stable process.) For symmetrical $\beta$-stable processes, (2) is clearly satisfied with $c_{0}=\beta$ and (a), (b), or (c) applied. Hence, for $\max (r, s)<\beta$ we obtain

$$
\Delta_{n}^{1-(r+s) / \beta} V_{r, s}\left(X, S_{n}\right) \stackrel{\mathrm{P}}{\rightarrow} \mu_{r, \beta} \mu_{s, \beta} \int_{0}^{t} \sigma_{u}^{(r+s) / \beta} \mathrm{d} u,
$$

as $n \rightarrow \infty$.

Example. (Generalized hyperbolic Lévy motion.) The generalized hyperbolic Lévy motion includes many processes used in finance. The density at $t=1$ is given by

$$
\begin{aligned}
& d_{G H(\lambda, \alpha, \gamma, \delta, \mu)}(x) \\
& \quad=a(\lambda, \alpha, \gamma, \delta, \mu)\left(\delta^{2}+(x-\mu)^{2}\right)^{(\lambda-1 / 2) / 2} \mathrm{e}^{\gamma(x-\mu)} K_{\lambda-1 / 2}\left(\alpha \sqrt{\delta^{2}+(x-\mu)^{2}}\right),
\end{aligned}
$$

where

$$
a(\lambda, \alpha, \gamma, \delta, \mu)=\frac{\left(\alpha^{2}-\gamma^{2}\right)^{\lambda / 2}}{\sqrt{2 \pi} \alpha^{\lambda-1 / 2} \delta^{\lambda} K_{\lambda}\left(\delta \sqrt{\alpha^{2}-\gamma^{2}}\right)},
$$

and $K_{\lambda}$ denotes the modified Bessel function of the third kind with index $\lambda$. We have the following parameter dependence, $\alpha>0$ determines the sharp, $0 \leq|\gamma|<\alpha$ the skewness, $\mu \in \mathbb{R}$ is a location parameter and $\delta>0$ determines the scaling. In general the density of the process is only known for $t=1$, however, we can give a formula for the Lévy triplet

$$
\left(t \mu, 0, t \frac{\mathrm{e}^{\gamma x}}{|x|}\left(\int_{0}^{\infty} \frac{\mathrm{e}^{-\sqrt{2 y+\alpha^{2}}|x|}}{\pi^{2} y\left(J_{|\lambda|}^{2}(\delta \sqrt{2 y})+Y_{|\lambda|}^{2}(\delta \sqrt{2 y})\right)} \mathrm{d} y+\max \{0, \lambda\} \mathrm{e}^{-\alpha|x|}\right)\right),
$$


where $J_{\lambda}$ denotes the Bessel function of the first order with index $\lambda$, and $Y_{\lambda}$ the Bessel function of the second order with index $\lambda$. Furthermore, we have $\alpha, \delta>0$, and $0 \leq|\gamma|<\alpha$. For $\lambda=1$ we obtain the hyperbolic Lévy motion, for $\lambda=-\frac{1}{2}$ the normal inverse Gaussian process and for $\alpha=\gamma=0$ the Student-t process. For a discussion of all possible limiting cases see Eberlein and von Hammerstein (2004).

From Raible (2000) we know that the density of the Lévy measure can be expanded in the form

$$
g(x)=\frac{\delta}{\pi x^{2}}+\frac{\lambda+\frac{1}{2}}{2} \frac{|x|}{x^{2}}+\frac{\delta \gamma}{\pi} \frac{1}{x}+o\left(\frac{|x|}{x^{2}}\right),
$$

as $x \rightarrow 0$. Furthermore, the first moment exists which is equivalent to $\int|x| 1_{|x|>1}(x) g(x)<\infty$. Hence, (2) and (b) are satisfied and for $p<1$ we obtain (3) with $\gamma=1$ and $c_{0}=\delta / \pi$. We see that we can consider all possible limiting cases for the parameters, except $\delta \rightarrow 0$ which would require changing the Blumenthal-Getoor index to zero.

Example. (CGMY process.) CGMY processes form a flexible class for modelling in finance, see, e.g. Carr et al. (2002). The density of the Lévy measure is given by

$$
\mathcal{C} \frac{\exp (-g|x|)}{|x|^{1+y}} 1_{x<0}(x)+\mathcal{C} \frac{\exp (-\mathcal{M}|x|)}{|x|^{1+y}} 1_{x>0}(x),
$$

where $\mathcal{C}>0$ describes the overall level of activity, $g, \mathcal{M} \geq 0$ control the rate of exponential decay on the right and the left tail, and $y<2$ characterizes the fine structure of the process. Clearly, for $2>y>0$ condition (2) is satisfied by Taylor expanding the exponential term. For $\mathcal{G}, \mathcal{M}>0$, (a) or (b) is satisfied, whereas for $\mathcal{G}=\mathcal{M}=0$ we are back to a stable process. Hence, we obtain (3) with $\beta=\mathcal{y}$ and $c_{0}=\mathcal{C}$.

The conditions on the mean process $Y$ are basically satisfied when $Y$ is dominated by $X$ in the sense that it possesses more regularity in the sample paths. Ait-Sahalia and Jacod (2007) introduced a similar concept of dominance when $X$ is a stable process. The process $Y$ can either be viewed as part of the model, e.g. as a continuous mean component of the form $\int_{0}^{t} a_{s} \mathrm{~d} s$ or a leverage component. Furthermore, it can be interpreted as a continuous market microstructure noise or market friction component, especially when we have a low jump frequency. For a discussion of market microstructure noise in a continuous semimartingale setting, see, e.g. Barndorff-Nielsen and Shephard (2007).

Example. (Hölder continuous process.) If $Y$ is Hölder continuous of the order $\gamma \in(1 / \beta, 1]$ the conditions on $Y$ are satisfied, as

$$
\begin{aligned}
& \Delta_{n}^{1-(r+s) / \beta} \sum_{i=1}^{n-1}\left|Y_{n, i+1}\right|^{r}\left|Y_{n, i}\right|^{s} \leq t C \Delta_{n}^{(r+s)(\gamma-1 / \beta)}, \\
& \Delta_{n}^{1-(r+s) / \beta} \sum_{i=1}^{n-1}\left|X_{n, i+1}\right|^{r}\left|Y_{n, i}\right|^{s} \leq C \Delta_{n}^{1-s / \beta+s \gamma} \sum_{i=1}^{n-1}\left|\frac{X_{n, i+1}}{\Delta_{n}^{1 / \beta}}\right|^{r},
\end{aligned}
$$

which both tend to zero as $n \rightarrow \infty$. Hence, we can only have a continuous mean component if $\beta>1$, which means that there are sufficiently many small jumps, i.e. the driving process is close to the behaviour of a Brownian motion. 
Example. (Compound Poisson process.) If $Y$ is a compound Poisson process the conditions are satisfied. For (4), we have only to note that by taking sufficiently large $n$ the probability of contiguous jumps is zero. For (5) we can use the fact that only finitely many terms contribute and $s<\beta$.

Example. (Infinite activity jump process.) One possibility is to take a jump process $Y$ having the same structure as $X$ with Blumenthal-Getoor index $\gamma<\beta$, then for $\max (r, s)<\gamma$ the conditions on $Y$ are satisfied using the technique discussed in Theorem 1. Of course, this leads to a restriction on the range of $r$ and $s$ which is not desirable from the point of view of estimating the squared integrated volatility with as few neighbouring increments as possible. However, putting $\beta \geq 1$ we can get rid of this restriction. Assume that $Y$ is some pure jump process with Blumenthal-Getoor index $0<\gamma<\beta$ and $\beta \geq 1$. The idea is to use the same technique as in Woerner (2003b), namely, splitting the process $Y$ into a component with finitely many jumps bigger than $\varepsilon$, for which we can argue as we did for the compound Poisson process, and a process with infinitely many small jumps. We define $I_{j}(\varepsilon)$ to be one if there are no jumps of absolute value bigger than $\varepsilon$ in the $j$ th time interval and zero otherwise. Furthermore, denote

$$
Y_{t}^{\varepsilon}=Y_{t}-\sum\left(J\left(Y_{s}\right):\left|J\left(Y_{s}\right)\right|>\varepsilon, 0<s \leq t\right),
$$

where $J\left(Y_{s}\right)=Y_{s}-Y_{s-}$, hence denote the jumps of $Y$. It is clear that $\left|Y_{j}\right|^{s} I_{j}(\varepsilon) \leq\left|Y_{j}^{\varepsilon}\right|^{s}$ and that for sufficiently small $\Delta_{n}$, $\sup _{j}\left|Y_{j}^{\varepsilon}\right| \leq 2 \varepsilon$ and $\Delta_{n} \leq 2 \varepsilon$. Hence, we can bound the part with the small jumps of (5), using Hölder's inequality with $1 / a+1 / b=1$, by

$$
\left(\Delta_{n}^{1-a r / \beta} \sum_{i=1}^{n-1}\left|X_{n, i+1}\right|^{a r}\right)^{1 / a}\left((2 \varepsilon)^{1-b s / \beta+b s-c} \sum_{i=1}^{n-1}\left|Y_{n, i}^{\varepsilon}\right|^{c}\right)^{1 / b}
$$

where $c \in(\gamma, b s)$. Choosing $a$ close to one, the first term tends to a finite limit as $r<\beta$ by (3). The second term tends to zero by first letting $n$ tend to infinity and then letting $\varepsilon$ tend to zero, provided that $b s>\gamma$ and $1-b s / \beta+b s-\gamma>0$. This is clearly satisfied if $\beta \geq 1$ and $\gamma<1$. For (4), the parts involving jumps bigger than $\varepsilon$ can be treated as the compound Poisson case, while the part with only small jumps can be treated using the same technique as for (5).

Next we derive a distributional theory, which is feasible as the variance term can also be estimated in terms of multipower variation estimates. Again, we look at the case of bipower variation first, though for the absolute integrated volatility it turns out that we need tripower and for the squared integrated volatility we need quintpower.

The conditions under which a mean process is negligible are a bit more restrictive than for consistency, namely, now no continuous mean process is possible. The form of the normalizing constant $C$ has a similar form as in the Brownian motion case and also shares the property that multipower variation is less efficient than power variation.

Theorem 3. Let

$$
X_{t}=L_{\int_{0}^{t} \sigma_{s} \mathrm{~d} s}
$$

Assume the same conditions on $L$ and $\sigma$ as in Theorem 1. Furthermore, we assume that $\sigma$ satisfies

$$
\sum_{i=1}^{n-1} \Delta_{n}^{1 / 2}\left(\left(\frac{\int_{i \Delta_{n}}^{(i+1) \Delta_{n}} \sigma_{u} \mathrm{~d} u}{\Delta_{n}}\right)^{r / \beta}\left(\frac{\int_{(i-1) \Delta_{n}}^{i \Delta_{n}} \sigma_{u} \mathrm{~d} u}{\Delta_{n}}\right)^{s / \beta}-\frac{\int_{(i-1) \Delta_{n}}^{i \Delta_{n}} \sigma_{u}^{(r+s) / \beta} \mathrm{d} u}{\Delta_{n}}\right) \rightarrow 0 .
$$


Then, for any $t>0$ and $\max (r, s)<\beta / 2$, we obtain

$$
\frac{\Delta_{n}^{1-(r+s) / \beta} V_{r, s}\left(X, S_{n}\right)-\mu_{r, \beta} \mu_{s, \beta}\left(c_{0} / \beta\right)^{(r+s) / \beta} \int_{0}^{t} \sigma_{u}^{(r+s) / \beta} \mathrm{d} u}{\sqrt{\mu_{2 r, \beta}^{-1} \mu_{2 s, \beta}^{-1} C \Delta_{n}^{2-2(r+s) / \beta} V_{2 r, 2 s}\left(X, S_{n}\right)}} \stackrel{\mathrm{D}}{\rightarrow} N(0,1),
$$

as $n \rightarrow \infty$, where $\mu_{p, \beta}=\mathrm{E}\left(|S|^{p}\right)$ with $S$ a symmetrical $\beta$-stable random variable and $C=\mu_{2 r, \beta} \mu_{2 s, \beta}+2 \mu_{r, \beta} \mu_{s, \beta} \mu_{r+s, \beta}-3 \mu_{r, \beta}^{2} \mu_{s, \beta}^{2}$.

We obtain the same result for $Z_{t}=Y_{t}+X_{t}$, when $Y_{t}$ is some stochastic process satisfying

$$
\begin{gathered}
\Delta_{n}^{1-2(r+s) / \beta} V_{2 r, 2 s}\left(Y, S_{n}\right) \stackrel{\mathrm{P}}{\rightarrow} 0, \\
\Delta_{n}^{1-2(r+s) / \beta} \sum_{i=1}^{n-1}\left|X_{(i+1) \Delta_{n}}-X_{i \Delta_{n}}\right|^{2 r}\left|Y_{i \Delta_{n}}-Y_{(i-1) \Delta_{n}}\right|^{2 s} \stackrel{\mathrm{P}}{\rightarrow} 0, \\
\Delta_{n}^{1 / 2-(r+s) / \beta} V_{r, s}\left(Y, S_{n}\right) \stackrel{\mathrm{P}}{\rightarrow} 0, \\
\Delta_{n}^{1 / 2-(r+s) / \beta} \sum_{i=1}^{n-1}\left|X_{(i+1) \Delta_{n}}-X_{i \Delta_{n}}\right|^{r}\left|Y_{i \Delta_{n}}-Y_{(i-1) \Delta_{n}}\right|^{s} \stackrel{\mathrm{P}}{\rightarrow} 0,
\end{gathered}
$$

where (19) and (21) also have to hold when $X$ and $Y$ are exchanged.

Proof. First of all, by (18) and (19) together with Theorem 1 and Slutzky's Lemma, to establish (17), it is sufficient to show

$$
\frac{\Delta_{n}^{1-(r+s) / \beta} V_{r, s}\left(X, S_{n}\right)-\mu_{r, \beta} \mu_{s, \beta}\left(c_{0} / \beta\right)^{(r+s) / \beta} \int_{0}^{t} \sigma_{u}^{(r+s) / \beta} \mathrm{d} u}{\Delta_{n}^{1 / 2} \sqrt{C\left(c_{0} / \beta\right)^{2(r+s) / \beta} \int_{0}^{t} \sigma_{u}^{2(r+s) / \beta} \mathrm{d} u}} \stackrel{\mathrm{D}}{\rightarrow} N(0,1) .
$$

Now we consider the case $Y=0$. We apply the central limit theorem (CLT) for triangular arrays (cf. Gnedenko and Kolmogorov (1968)), which states that for independent $\chi_{n, i}$ with $\mathrm{E}\left(\chi_{n, i}\right)=0$, if for $n \rightarrow \infty$,

$$
\begin{aligned}
& \sum_{i=1}^{n} \mathrm{E}\left(\chi_{n, i}^{2}\right) \rightarrow S^{2}>0 \\
& \sum_{i=1}^{n} \mathrm{E}\left(\chi_{n, i}^{2} 1_{\left|\chi_{n, i}\right|>\varepsilon}\right) \rightarrow 0
\end{aligned}
$$

for all $\varepsilon>0$, then

$$
\sum_{i=1}^{n} \chi_{n, i} \stackrel{\mathrm{D}}{\rightarrow} N\left(0, S^{2}\right)
$$

To get independent increments we have to use the same method as in Theorem 1, working conditionally under $\sigma$ and rearranging to independent copies of $\sum_{i=1}^{n} \chi_{n, i}$. For the rearranged summands, we set

$$
\tilde{\chi}_{n, i}=\Delta_{n}^{1 / 2}\left(\left|\frac{\tilde{X}_{n, i+1}}{\Delta_{n}^{1 / \beta}}\right|^{r}\left|\frac{\tilde{X}_{n, i}}{\Delta_{n}^{1 / \beta}}\right|^{s}-\mathrm{E}\left[\left|\frac{\tilde{X}_{n, i+1}}{\Delta_{n}^{1 / \beta}}\right|^{r}\left|\frac{\tilde{X}_{n, i}}{\Delta_{n}^{1 / \beta}}\right|^{s}\right]\right) .
$$


Clearly (23) is satisfied, as $2(r+s)<\beta$. For (22), we have to use (6) and (10). This yields that the sum of the two independent copies of $\sum_{i=1}^{n} \chi_{n, i}$ is normally distributed, hence, $\sum_{i=1}^{n} \chi_{n, i}$ is also normally distributed and we only have to calculate the appropriate variance. As we have correlations between neighbouring terms, we obtain the constant

$$
C=\mu_{2 r, \beta} \mu_{2 s, \beta}+2 \mu_{r, \beta} \mu_{s, \beta} \mu_{r+s, \beta}-3 \mu_{r, \beta}^{2} \mu_{s, \beta}^{2},
$$

and the variance

$$
S^{2}=C\left(\frac{c_{0}}{\beta}\right)^{2(r+s) / \beta} \int_{0}^{t} \sigma_{u}^{2(r+s) / \beta} \mathrm{d} u
$$

Altogether, this yields

$$
\sum_{i=1}^{n-1} \frac{\Delta_{n}^{1 / 2}}{S}\left(\left|\frac{X_{n, i+1}}{\Delta_{n}^{1 / \beta}}\right|^{r}\left|\frac{X_{n, i}}{\Delta_{n}^{1 / \beta}}\right|^{s}-\mathrm{E}\left[\left|\frac{X_{n, i+1}}{\Delta_{n}^{1 / \beta}}\right|^{r}\left|\frac{X_{n, i}}{\Delta_{n}^{1 / \beta}}\right|^{s}\right]\right) \stackrel{\mathrm{D}}{\rightarrow} N(0,1) .
$$

Hence, it remains to show that in probability as $n \rightarrow \infty$,

$$
\sum_{i=1}^{n-1} \Delta_{n}^{1 / 2} \mathrm{E}\left[\left|\frac{X_{n, i+1}}{\Delta_{n}^{1 / \beta}}\right|^{r}\left|\frac{X_{n, i}}{\Delta_{n}^{1 / \beta}}\right|^{s} \mid \sigma\right]-\Delta_{n}^{-1 / 2} \mu_{r, \beta} \mu_{s, \beta}\left(\frac{c_{0}}{\beta}\right)^{(r+s) / \beta} \int_{0}^{t} \sigma_{u}^{(r+s) / \beta} \mathrm{d} u \rightarrow 0 .
$$

This follows from

$$
\sum_{i=1}^{n-1} \Delta_{n}^{1 / 2}\left(\mathrm{E}\left[\left|\frac{X_{n, i+1}}{\Delta_{n}^{1 / \beta}}\right|^{r}\left|\frac{X_{n, i}}{\Delta_{n}^{1 / \beta}}\right|^{s} \mid \sigma\right]-\mu_{r, \beta} \mu_{s, \beta}\left(\frac{c_{0}}{\beta}\right)^{(r+s) / \beta} \sigma_{n, i}^{r+s / \beta}\right) \rightarrow 0,
$$

as

$$
\mathrm{E}\left[\left|\frac{X_{n, i+1}}{\Delta_{n}^{1 / \beta}}\right|^{r}\left|\frac{X_{n, i}}{\Delta_{n}^{1 / \beta}}\right|^{s} \mid \sigma\right]-\mu_{r, \beta} \mu_{s, \beta}\left(\frac{c_{0}}{\beta}\right)^{(r+s) / \beta} \sigma_{n, i-1}^{r / \beta} \sigma_{n, i}^{s / \beta}=O\left(\Delta_{n}^{1 / \beta}\right),
$$

by (6) and furthermore

$$
\sum_{i=1}^{n-1} \Delta_{n}^{1 / 2}\left(\left(\frac{\int_{i \Delta_{n}}^{(i+1) \Delta_{n}} \sigma_{u} \mathrm{~d} u}{\Delta_{n}}\right)^{r / \beta}\left(\frac{\int_{(i-1) \Delta_{n}}^{i \Delta_{n}} \sigma_{u} \mathrm{~d} u}{\Delta_{n}}\right)^{s / \beta}-\frac{\int_{(i-1) \Delta_{n}}^{i \Delta_{n}} \sigma_{u}^{(r+s) / \beta} \mathrm{d} u}{\Delta_{n}}\right) \rightarrow 0,
$$

by assumption (16). Again the unconditional version of the CLT follows straightforwardly from the conditional one.

Next we have to look at the case with nontrivial mean process $Y$. By Slutzky's Lemma we only have to establish that

$$
\Delta_{n}^{1 / 2-(r+s) / \beta}\left(V_{r, s}\left(X+Y, S_{n}\right)-V_{r, s}\left(X, S_{n}\right)\right) \stackrel{\mathrm{P}}{\rightarrow} 0,
$$

as $n \rightarrow \infty$. As in Theorem 1 this holds if

$$
\begin{gathered}
\Delta_{n}^{1 / 2-(r+s) / \beta} V_{r, s}\left(Y, S_{n}\right) \stackrel{\mathrm{P}}{\rightarrow} 0, \\
\Delta_{n}^{1 / 2-(r+s) / \beta} \sum_{i=1}^{n-1}\left|X_{(i+1) \Delta_{n}}-X_{i \Delta_{n}}\right|^{r}\left|Y_{i \Delta_{n}}-Y_{(i-1) \Delta_{n}}\right|^{s} \stackrel{\mathrm{P}}{\rightarrow} 0,
\end{gathered}
$$

which are (20) and (21), respectively. This completes our proof. 
Again we have to look at examples, when the conditions on $Y$ are satisfied.

Example. (Compound Poisson process.) The same arguments as for Theorem 1 together with $\max (r, s)<\beta / 2$ yield that (18) to (21) are satisfied.

Example. (Infinite activity jumps.) We take a pure jump process $Y$ with Blumenthal-Getoor index $\gamma, \gamma<\beta$ and $\beta \geq 1$. Condition (18) and (19) are satisfied as (4) and (5), noting that now $\max (r, s)<\beta / 2$.

For (20) and (21), we use the same technique as before, splitting the process in a part with small jumps and a compound Poisson part, for which the result is clear. Hence, it remains to look at the part with jumps smaller than $\varepsilon$. Using Hölder's inequality with $1 / a+1 / b+1 / c=1$, we can bound the part with small jumps of (20) by

$$
\begin{gathered}
\left(\sum_{i=1}^{n-1} \Delta_{n}\right)^{1 / a}\left((2 \varepsilon)^{1-b / 4-b r / \beta+b r-d} \sum_{i=1}^{n-1}\left|Y_{n, i+1}^{\varepsilon}\right|^{r b}\right)^{1 / b} \\
\times\left((2 \varepsilon)^{1-c / 4-c s / \beta+c s-e} \sum_{i=1}^{n-1}\left|Y_{n, i}^{\varepsilon}\right|^{c s}\right)^{1 / c}
\end{gathered}
$$

where $d \in(\gamma, b r)$ and $e \in(\gamma, c s)$. The second term of the upper bound tends to zero if $b r>\gamma$ and $1-b / 4-b r / \beta+b r-\gamma>0$, which leads to

$$
r>\frac{\gamma}{4(1-\gamma / \beta)},
$$

and similarly for the third term. Analogous considerations for (21) lead to

$$
r, s>\frac{\gamma}{2(1-\gamma / \beta)} .
$$

Summarizing the result $Y$ is negligible if it is a pure jump process with Blumenthal-Getoor index $\gamma, \gamma<\beta, \beta \geq 1$, and the exponents satisfy $\max (r, s)<\beta / 2$ and

$$
\min (r, s)>\frac{\gamma}{2(1-\gamma / \beta)}
$$

Finally we have to look at condition (16). Obviously it is satisfied if $\sigma$ is Hölder continuous of the order $\gamma \in\left(\frac{1}{2}, 1\right]$, i.e. satisfied by a fractional Brownian motion with Hurst exponent $H>\frac{1}{2}$. Fortunately the conditions are not as restrictive as they look at first glance. Using BarndorffNielsen et al. (2006a) for the special case that $\sigma$ is independent of the driving Brownian motion, their equations (7.2) and (7.1) reduce to

$$
\begin{gathered}
\sum_{i=1}^{n} \Delta_{n}^{1 / 2} \frac{\int_{(i-1) \Delta_{n}}^{i \Delta}\left(\sigma_{u}^{p}-\sigma_{(i-1) \Delta_{n}}^{p}\right) \mathrm{d} u}{\Delta_{n}} \rightarrow 0, \\
\sum_{i=1}^{n-1} \Delta_{n}^{1 / 2}\left(\left|\frac{\int_{i \Delta_{n}}^{(i+1) \Delta} \sigma_{u}^{2} \mathrm{~d} u}{\Delta_{n}}\right|^{r / 2}\left|\frac{\int_{(i-1) \Delta_{n}}^{i \Delta} \sigma_{u}^{2} \mathrm{~d} u}{\Delta_{n}}\right|^{s / 2}-\sigma_{(i-1) \Delta_{n}}^{r+s}\right) \rightarrow 0,
\end{gathered}
$$

respectively for some $p, r, s>0$. This implies condition (16) by inserting plus and minus $\sigma_{(i-1) \Delta_{n}}^{r+s / \beta}$ and using triangular inequality. Hence, condition (16) is satisfied if $\sigma$ and $\sigma^{2}$ are 
semimartingales of the form

$$
\begin{aligned}
\sigma_{t}= & \sigma_{0}+\int_{0}^{t} b_{s} \mathrm{~d} s+\int_{0}^{t} c_{s-} \mathrm{d} W_{s}+\int_{0}^{t} \int_{E} h \circ w(s, x)(\mu-v)(\mathrm{d} s, \mathrm{~d} x) \\
& +\int_{0}^{t} \int_{E}(w-h \circ w(s, x)) \mu(\mathrm{d} s, \mathrm{~d} x)
\end{aligned}
$$

where $W$ is a Brownian motion and $\mu$ a Poisson random independent of $W$, for more details cf. Barndorff-Nielsen et al. (2006a). This condition especially holds for the cases of diffusion processes, e.g. the Cox-Ingersoll-Ross process, or jump processes, e.g. Ornstein-Uhlenbecktype processes.

As for consistency we get an analogous distributional result for the multipower variation case, which makes it possible to estimate even higher powers of the volatility.

Theorem 4. Let

$$
X_{t}=L_{\int_{0}^{t} \sigma_{s} \mathrm{~d} s} .
$$

Assume the same conditions on $L$ and $\sigma$ as in Theorem 1. Furthermore, we assume that $\sigma$ satisfies

$$
\sum_{i=1}^{n-k+1} \Delta_{n}^{1 / 2}\left(\prod_{j=1}^{k}\left(\frac{\int_{(i-1+j) \Delta_{n}}^{(i+j) \Delta_{n}} \sigma_{u} \mathrm{~d} u}{\Delta_{n}}\right)^{r_{j} / \beta}-\frac{\int_{(i-1) \Delta_{n}}^{i \Delta_{n}} \sigma_{u}^{\sum_{j=1}^{k} r_{j} / \beta} \mathrm{d} u}{\Delta_{n}}\right) \rightarrow 0 .
$$

Then, for any $t>0$ and $\max _{1 \leq j \leq k}\left(r_{j}\right)<\beta / 2$, we obtain

$$
\frac{\Delta_{n}^{1-\sum_{j=1}^{k} r_{j} / \beta} V_{r_{1}, \ldots, r_{k}}\left(X, S_{n}\right)-\prod_{j=1}^{k} \mu_{r_{j}, \beta}\left(c_{0} / \beta\right)^{\sum_{j=1}^{k} r_{j} / \beta} \int_{0}^{t} \sigma_{u}^{\sum_{j=1}^{k} r_{j} / \beta} \mathrm{d} u}{\sqrt{\prod_{j=1}^{k} \mu_{2 r_{j}, \beta}^{-1} C \Delta_{n}^{2-2 \sum_{j=1}^{k} r_{j} / \beta} V_{2 r_{1}, \ldots, 2 r_{k}}\left(X, S_{n}\right)}} \stackrel{\mathrm{D}}{\rightarrow} N(0,1),
$$

as $n \rightarrow \infty$, where $\mu_{p, \beta}=\mathrm{E}\left(|S|^{p}\right)$ with $S$ a symmetrical $\beta$-stable random variable and

$$
C=\prod_{l=1}^{k} \mu_{2 r_{l}, \beta}-(2 k-1) \prod_{l=1}^{k} \mu_{r_{l}, \beta}^{2}+2 \sum_{i=1}^{k-1} \prod_{l=1}^{i} \mu_{r_{l}, \beta} \prod_{l=k-i+1}^{k} \mu_{r_{l}, \beta} \prod_{l=1}^{k-i} \mu_{r_{l}+r_{l+i}, \beta} .
$$

Proof. The proof follows the same lines as the proof of Theorem 3. The formula for $C$ may be derived analogously as in Barndorff-Nielsen et al. (2006a).

\section{Applications to different models}

In the following we will study how we can apply the general result to some specific Lévy-type stochastic volatility models, most of which were introduced in Carr et al. (2003). As driving Lévy processes we consider the three most popular Lévy processes in finance: the NIG, the hyperbolic Lévy process, and the CGMY process, whereas as volatility process we consider a mean-reverting process, e.g. a Cox-Ingersoll-Ross process or an Ornstein-Uhlenbeck-type process driven by a jump process or a fractional Brownian motion with Hurst exponent $H>\frac{1}{2}$. The latter case was studied in the framework of classical stochastic volatility models by Comte and Renault (1998) introducing long memory. 
We state only the results for the most popular cases, $\int_{0}^{t} \sigma_{u}^{p} \mathrm{~d} u$ for $p=1$ or $p=2$. However, general $p$ can be treated analogously. Furthermore, we will choose the estimators with equal power coefficients. As before we always have to assume that the volatility process and the driving Lévy process are independent, which is the same setting as in Carr et al. (2003).

\subsection{NIG based models}

First of all we look at models of the form $X_{t}=L_{\int_{0}^{t} \sigma_{u} \mathrm{~d} u}$ where $L$ is NIG. Clearly, the Blumenthal-Getoor index is $\beta=1$ and the constant $c_{0}=\delta / \pi$ by (15). Hence, we obtain

$$
\begin{gathered}
\frac{V_{1 / 2,1 / 2}\left(X, S_{n}\right)}{\left(\mu_{1 / 2,1}\right)^{2} \delta / \pi} \stackrel{\mathrm{P}}{\rightarrow} \int_{0}^{t} \sigma_{u} \mathrm{~d} u, \\
\frac{V_{2 / 3,2 / 3,2 / 3}\left(X, S_{n}\right)}{\Delta_{n}\left(\mu_{2 / 3,1}\right)^{3}(\delta / \pi)^{2}} \stackrel{\mathrm{P}}{\rightarrow} \int_{0}^{t} \sigma_{u}^{2} \mathrm{~d} u .
\end{gathered}
$$

These results hold for all three types of volatility processes. For the distributional theory we need a higher order of the multipower, namely

$$
\begin{aligned}
& \frac{V_{1 / 3,1 / 3,1 / 3}\left(X, S_{n}\right)-\left(\mu_{1 / 3,1}\right)^{3} \delta / \pi \int_{0}^{t} \sigma_{u} \mathrm{~d} u}{\sqrt{\left(\mu_{2 / 3,1}\right)^{-3} C_{1} V_{2 / 3,2 / 3,2 / 3}\left(X, S_{n}\right)}} \stackrel{\mathrm{D}}{\rightarrow} N(0,1), \\
& \frac{\Delta_{n}^{-1} V_{2 / 5,2 / 5,2 / 5,2 / 5,2 / 5}\left(X, S_{n}\right)-\left(\mu_{2 / 5,1}\right)^{5}(\delta / \pi)^{2} \int_{0}^{t} \sigma_{u}^{2} \mathrm{~d} u}{\Delta_{n}^{-1} \sqrt{\left(\mu_{4 / 5,1}\right)^{-5} C_{2} V_{4 / 5,4 / 5,4 / 5,4 / 5,4 / 5}\left(X, S_{n}\right)}} \stackrel{\mathrm{D}}{\rightarrow} N(0,1),
\end{aligned}
$$

where

$$
C_{1}=\left(\mu_{2 / 3,1}\right)^{3}-5\left(\mu_{1 / 3,1}\right)^{6}+2\left(\mu_{1 / 3,1}\right)^{2}\left(\mu_{2 / 3,1}\right)^{2}+2\left(\mu_{1 / 3,1}\right)^{4} \mu_{2 / 3,1},
$$

and

$$
C_{2}=\left(\mu_{4 / 5,1}\right)^{5}-9\left(\mu_{2 / 5,1}\right)^{10}+2 \sum_{i=1}^{4}\left(\mu_{2 / 5,1}\right)^{2 i}\left(\mu_{4 / 5,1}\right)^{5-i} .
$$

Changing the model to include leverage, our method works when the volatility process is an OU-type process. Looking at

$$
\begin{gathered}
X_{t}=\rho Z_{t}+L_{\int_{0}^{t} \sigma_{u} \mathrm{~d} u}, \\
d \sigma_{t}=-\kappa \sigma_{t} d s+d Z_{t},
\end{gathered}
$$

we get (26) and (27) independent of which one-sided Lévy process drives the volatility process. For the distributional theory we have to take into account that by (24) we also get a lower bound on the possible power exponents. Hence, (28) still holds if $Z$ is a Gamma process or a compound Poisson process, which both have Blumenthal-Getoor index zero. For (29), our result holds in addition for $Z$ being an inverse Gaussian (IG) process, which has Blumenthal-Getoor index $\frac{1}{2}$.

\subsection{Hyperbolic Lévy motion based models}

In this case we get exactly the same results as for the NIG case. In fact the same result holds for all processes of the class of generalized hyperbolic processes, as by (15) they have all the same fine structure which determines the behaviour of the integrated volatility. 


\subsection{CGMY based models}

In this class of models the estimators depend on the size of $\mathcal{y}$, which is the BlumenthalGetoor index. Furthermore, the constant is $c_{0}=\mathcal{C}$. Again we will look at the model $X_{t}=L_{\int_{0}^{t} \sigma_{u} \mathrm{~d} u}$ first, where $L$ is now a CGMY process. We have to distinguish between the cases $y>1$ and $y \leq 1$.

For the consistency we obtain, for $1<y<2$,

$$
\begin{gathered}
\frac{V_{y}\left(X, S_{n}\right)}{\mu y, y(\mathcal{C} / \mathcal{Y})} \stackrel{\mathrm{P}}{\rightarrow} \int_{0}^{t} \sigma_{u} \mathrm{~d} u, \\
\frac{V y, y\left(X, S_{n}\right)}{\Delta_{n}(\mu y, y)^{2}(\mathcal{C} / \mathcal{y})^{2}} \stackrel{\mathrm{P}}{\rightarrow} \int_{0}^{t} \sigma_{u}^{2} \mathrm{~d} u,
\end{gathered}
$$

and, for $0<y \leq 1$,

$$
\begin{gathered}
\frac{V_{y / 2, y / 2}\left(X, S_{n}\right)}{(\mu y / 2, y)^{2}(\mathcal{C} / y)} \stackrel{\mathrm{P}}{\rightarrow} \int_{0}^{t} \sigma_{u} \mathrm{~d} u, \\
\frac{V_{2 y / 3,2 y / 3,2 y / 3}\left(X, S_{n}\right)}{\Delta_{n}\left(\mu_{2 y / 3, y)^{3}(\mathcal{C} / \mathcal{y})^{2}}\right.} \stackrel{\mathrm{P}}{\rightarrow} \int_{0}^{t} \sigma_{u}^{2} \mathrm{~d} u,
\end{gathered}
$$

and for the distributional theory, for $1<y<2$,

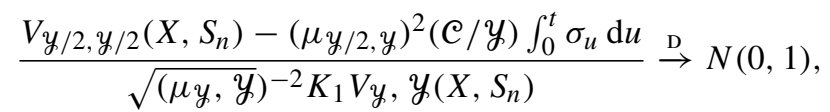

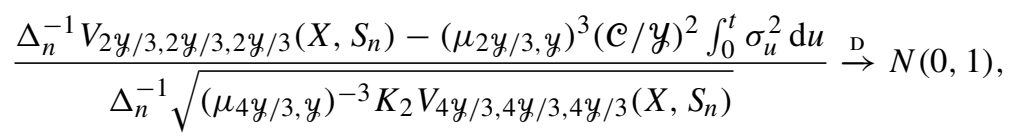

and, for $0<y \leq 1$,

$$
\begin{aligned}
& \frac{V y / 3, y / 3, y / 3}{\sqrt{\left(\mu_{2} y / 3, y\right)^{-3} K_{3} V_{2 y / 3,2 y / 3,2 y / 3}\left(X, S_{n}\right)}} \stackrel{\mathrm{D}}{\rightarrow} N(0,1),
\end{aligned}
$$

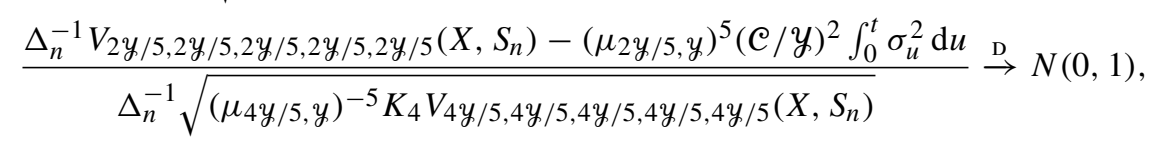

where $K_{1}$ to $K_{4}$ are calculated according to (25). This result holds for all three types of volatility processes.

The model with leverage may be treated as in the NIG case, only we have to take into account the fact that for infinite activity mean processes we need a Blumenthal-Getoor index of $\beta \geq 1$. Our result holds for $y \geq 1$ if the driving process $Z$ is a Gamma or a compound Poisson process. Furthermore the IG process may be used as a driving process for the estimate of $\int_{0}^{t} \sigma_{u} \mathrm{~d} u$ if $y>1$ and for the estimate of $\int_{0}^{t} \sigma_{u}^{2} \mathrm{~d} u$ if $y \geq 1$.

\section{Conclusion}

We have introduced the class of multipower variation estimators for Lévy-type stochastic volatility models. They lead to easily computable consistent estimators possessing a feasible 
distributional theory. We have shown that these estimators may be used to obtain estimates for the integrated volatility, especially absolute value and squares, based on high-frequency data. This method works for a large variety of models based on Lévy processes, such as NIG, CGMY, and hyperbolic ones, including models with leverage and allowing for market friction with lower activity than the driving process.

\section{Acknowledgement}

I would like to thank the referee for helpful comments and suggestions.

\section{References}

Ait-Sahalia, Y. and Jacod, J. (2007). Volatility estimators for discretely sampled Lévy processes. To appear in Ann. Statist.

Barndorff-Nielsen, O. E. et al. (2006a). A central limit theorem for realised power and bipower variations of continuous semimartingales. In From Stochastic Analysis to Mathematical Finance, eds Y. Kabanov, R. Lipster and J. Stoyanov, Springer, Berlin, pp. 33-68.

Barndorff-Nielsen, O. E. and Shephard, N. (2001). Non-Gaussian Ornstein-Uhlenbeck-based models and some of their uses in financial economics (with discussion). J. Roy. Statist. Soc. Ser. B 63, 167-241.

Barndorff-Nielsen, O. E. and Shephard, N. (2003). Realised power variation and stochastic volatility models. Bernoulli 9, 243-265.

Barndorff-Nielsen, O. E. and Shephard, N. (2004). Power and bipower variation with stochastic volatility and jumps. J. Financial Econometrics 2, 1-48.

Barndorff-Nielsen, O. E. and Shephard, N. (2005). Power variation and time change. Theory Prob. Appl. 50, 1-15.

Barndorff-Nielsen, O. E. and Shephard, N. (2006). Econometrics of testing for jumps in financial econometrics using bipower variation. J. Financial Econometrics 4, 1-30.

Barndorff-Nielsen, O. E. and Shephard, N. (2007). Variation, jumps, market frictions and high frequency data in financial econometrics. To appear in Advances in Economics and Econometrics, Theory and Applications, Ninth World Congress, Cambridge University Press.

Barndorff-Nielsen, O. E., Shephard, N. and Winkel, M. (2006b). Limit theorems for multipower variation in the presence of jumps. Stoch. Process. Appl. 116, 796-806.

Berman, S. M. (1965). Sign-invariant random variables and stochastic processes with sign invariant increments. Trans. Amer. Math. Soc. 119, 216-243.

Carr, P., Geman, H., Madan, D. B. and Yor, M. (2002). The fine structure of asset returns: an empirical investigation. J. Business 75, 305-332.

Carr, P., Geman, H., Madan, D. B. and Yor, M. (2003). Stochastic volatility for Lévy processes. Math. Finance 13, 345-382.

Comte, F. and Renault, E. (1998). Long memory in continuous-time stochastic valatility models. Math. Finance 8, 291-323.

Corcuera, J. M., Nualart, D. and Woerner, J. H. C. (2006). Power variation of some integral fractional processes. Bernoulli 12, 713-735.

Corcuera, J. M., Nualart, D. and Woerner, J. H. C. (2007). A functional central limit theorem for the realized power variation of integrated stable processes. Stoch. Anal. Appl. 25, 169-186.

Eberlein, E. and von Hammerstein, E. A. (2004). Generalized hyperbolic and inverse Gaussian distributions: limiting cases and approximation of processes. Seminar on Stochastic Analysis, Random Fields and Applications IV (Progress. Prob. 58), Birkhäuser, Basel, pp. 221-264.

Geman, H., Madan, D. B. and Yor, M. (2001). Time changes for Lévy processes. Math. Finance 11, 79-96.

Gnedenko, B. V. and Kolmogorov, A. N. (1968). Limit Distributions for Sums of Independent Random Variables. Addison-Wesley, Reading, MA.

Howison, S., Rafailidis, A. and Rasmussen, H. O. (2002). A note on the pricing and hedging of volatility derivatives. Tech. Rep. 2001-MF-09, OCIAM, University of Oxford.

Hudson, W. N. and Mason, J. D. (1976). Variational sums for additive processes. Proc. Amer. Math. Soc. 55, 395-399.

Lepingle, D. (1976). La variation d'ordre p des semi-martingales. Z. Wahrscheinlichkeitsth. 36, 295-316.

Raible, S. (1999). Lévy processes in finance: Theory, numerics, and empirical facts. PhD thesis, University of Freiburg, 2000.

Sato, K. (1999). Lévy Processes and Infinitely Divisible Distributions. Cambridge University Press. 
Woerner, J. H. C. (2003a). Purely discontinuous Lévy processes and power variation: inference for integrated volatility and the scale parameter. 2003-MF-07, Working Paper, University of Oxford.

Woerner, J. H. C. (2003b). Variational sums and power variation: a unifying approach to model selection and estimation in semimartingale models. Statist. Decisions 21, 47-68.

Woerner, J. H. C. (2005). Estimation of integrated volatility in stochastic volatility models. Appl. Stochastic Models Bus. Ind., 21:27-44.

Woerner, J. H. C. (2006). Power and multipower variation: inference for high frequency data. In Stochastic Finance, eds A. N. Shiryaev, M. do Rosário Grossihno, P. Oliviera, and M. Esquivel. Springer, New York, pp. 343-364. 